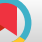

\title{
Pulsed Radiofrequency of the Occipital Nerves: Results of a Standardized Protocol on Chronic Headache Management
}

\author{
Chrysanthi Batistaki ${ }^{1}{ }^{1,}$, Alia Ibrahim Madi ${ }^{1}$, Agathi Karakosta ${ }^{1}$, Georgia Kostopanagiotou ${ }^{1}$ and Chrysa \\ Arvaniti $^{2}$ \\ ${ }^{1}$ 2nd Department of Anaesthesiology, School of Medicine, National and Kapodistrian University of Athens, Attikon Hospital, Athens, Greece \\ ${ }^{2}$ 2nd Department of Neurology, School of Medicine, National and Kapodistrian University of Athens, Attikon Hospital, Athens, Greece \\ "Corresponding author: 2nd Department of Anaesthesiology, School of Medicine, National and Kapodistrian University of Athens, Attikon Hospital, Athens, Greece. Email: \\ chrysabatistaki@yahoo.gr
}

Received 2021 January 03; Revised 2021 August 28; Accepted 2021 August 30.

\begin{abstract}
Background: Pulsed radiofrequency (PRF) of the occipital nerves has neuromodulative properties and is used for chronic pain management. However, its role in various types of chronic headaches has not been adequately investigated so far.

Objectives: This was an observational, open-label, prospective study aiming to assess the efficacy of PRF of occipital nerves on various types of chronic headache management.

Methods: Patients with chronic headaches followed up at the pain management unit were scheduled for PRF of both occipital nerves after a positive diagnostic nerve block. PRF was applied following a standardized protocol at $42^{\circ} \mathrm{C}$, and the number of headaches per month was assessed as a primary outcome at baseline (before treatment), as well as after 1, 3, and 6 months. Pain intensity during headache crises was recorded using the Numeric Rating Scale (NRS, 0 - 10),

Results: Fifty-seven patients suffering from chronic migraines, cluster headaches, tension-type headaches, and occipital neuralgia were studied. PRF significantly improved the number of headache episodes per month, as well as the pain intensity of the crises. The median number of headache episodes per month was significantly reduced in patients with migraine, from 14.5 to 4 after 1 month, and to 6.5 after 6 months. The same was seen for patients with clusters, who were also improved. A statistically significant reduction in NRS values over time was seen for all types of headaches.

Conclusions: PRF of the occipital nerves can lead to a reduction of the number of headache episodes per month, improving the intensity of pain during each episode.
\end{abstract}

Keywords: Headache, Pulsed Radiofrequency, Occipital Nerves

\section{Background}

Pulsed radiofrequency (PRF) of the greater (GON) and lesser (LON) occipital nerves is a minimally-invasive technique indicated for the management of chronic pain, of variable etiology, including headaches $(1,2)$. It has been used for chronic migraine, tension-type, and cluster headaches, as well as for cases of occipital neuralgia (1-4). The application of the technique follows standardized criteria and a previous positive diagnostic block of the nerves with local anesthetic $(1,5-8)$.

PRF exhibits a known neuromodulative effect due to its non-destructive properties, with a final temperature of the active tip not exceeding $42^{\circ} \mathrm{C}$ (9). It acts through an electric field of low intensity, which leads to a decreased conduction through the pathways of pain. Its main effect is through the unmyelinated C-fibers and not on myelinated ones $(1,2,10)$. Research showed that PRF significantly mod- ulates synaptic transmission, leading to long-term analgesia, and facilitates the inhibitory effect of serotonergic, noradrenergic, and endogenous opioid pain pathways $(2,10)$.

Although PRF has been studied in various types of chronic pain, its results on chronic headaches has not been adequately investigated.

\section{Objectives}

The aim of this study was to assess the efficacy of PRF of the occipital nerves on chronic headache management.

\section{Methods}

All subjects were patients of a Pain Management Unit of a University Hospital, suffering from chronic headaches (migraine, cluster, and tension type headache) 
as well as occipital neuralgia. The patients signed a written informed consent and were scheduled for interventional therapy, following the standard therapeutic protocol for headache management, including neurological examination, pharmacotherapy, and diagnostic nerve blocks. All patients were on prophylaxis with topiramate or amitriptyline, and used triptans for rescue treatment. Inclusion criteria were not adequate response to pharmacotherapy, pain during crises $>4 / 10$, occipital tenderness bilaterally/laterally, and days with headache $>$ $10 /$ month. Exclusion criteria were $<18$ years of age, pregnancy/lactation, a cardiac pacemaker, previous therapy with PRF at the same area, severe psychiatric diseases, coagulation disorders, and patient refusal. In cases of a positive response to the diagnostic nerve block with local anesthetic, with $>50 \%$ pain relief, patients were scheduled for PRF of GON/LON bilaterally, except for cases of occipital neuralgia, where treatment was performed unilaterally.

The procedure was performed with Diros RF hybrid cannula (22G, $54 \mathrm{~mm}, 4 \mathrm{~mm}$ active tip). At first, the two GONs were stimulated, and the LONs were stimulated (40 - 60V, $2 \mathrm{~Hz}$, impedance 150 - 400 $\Omega$, plateau temperature $\left.42^{\circ} \mathrm{C}, 6 \mathrm{~min}\right)$. Nerves were located using the classic anatomical landmarks for the technique, described in literature (4). The correct position of the needle was confirmed with mild stimulation and minimal adjustments until the optimum position was reached (optimum stimulation with $<$ $0.4 \mathrm{mV}$ ). Postoperatively, patients were allowed to recover for 2 hours and then discharged.

The primary outcome of the study was the difference in the number of headache episodes per month before and after treatment. Pain intensity during headache crisis (Numeric Rating Scale; NRS, 0 - 10) was studied secondarily. Analgesic consumption (number of analgesics per month) was also recorded. All measurements were performed before treatment, as well as after 1, 3, and 6 months. Patients completed the Greek edition of Brief Pain Inventory. All measurements were accomplished by a blinded researcher of the Pain Management Unit. All complications and side effects were recorded.

\subsection{Statistical Analysis}

The Shapiro-Wilk test was applied to test the normal distribution of continuous variables. The results are provided as mean \pm standard deviation (SD), or as median and interquartile range (IQR) according to normality. All qualitative variables are presented as absolute or relative frequencies. One-way analysis of variance (ANOVA) or Kruskal-Wallis test were applied to compare continuous variables and the Fisher's comparison of categorical variables. A repeated measures ANOVA model was performed to detect differences in mean NRS scores over time. The Bonferroni correction was used for multiple comparisons; all tests were two-tailed. Statistical significance was set at 5\% $(\mathrm{P}<0.05)$. Data were analyzed using Stata ${ }^{\mathrm{TM}}(10.1 \mathrm{MP}$, Stata Corporation, College Station, TX77845, USA).

\section{Results}

Fifty-seven patients were studied. Demographic characteristics of patients, as well as the type of headache, are presented in Table 1. Most of patients suffered from migraine $(n=38)$, while only nine patients suffered from cluster or other forms of headache (tension-type and occipital neuralgia). The median number of headache episodes per month was significantly reduced in patients with migraine, from 14.5 to 4 after 1 month, and to 6.5 after 6 months. The same was seen for patients with clusters, who were also improved (Tables 2 and 3; Figure 1). The repeated measures ANOVA model revealed a statistically significant reduction in pain intensity measured with NRS for all types of headaches (Table 4). Specifically, for each month, NRS values were reduced by an average of $0.28(\mathrm{P}<0.001)$ (Figure 2; Table 4). However, NRS comparisons between the study groups (migraine vs. cluster; migraine vs. other types of headaches) did not show any statistical significance.

Systemic pharmacotherapy was not different in different time points. Moreover, rescue treatment was not different between the groups (Table 5).

\section{Discussion}

PRF has been widely used on multiple targets with different outcomes on chronic pain conditions, such as peripheral neuralgias, trigeminal neuralgia, chronic knee pain, etc. $(2,3)$. Its results depend on the type of pain (aiming mostly on pain of neuropathic origin), type of the targeted nerves, duration of pain, technical characteristics of the devices used (such as the length of the active tip), time of PRF application, and more. However, the effect of PRF on chronic headaches has not been adequately studied. Most studies on the subject refer to occipital neuralgia only or combined with migraine, and there are not large case series or randomized clinical trials (RCTs) describing the effect of PRF only on migraines, cluster, or tension-type headaches. Hence, in this study, we focused on patients with chronic headaches, mostly migraines. The results showed that PRF of both occipital nerves had a favorable impact on patients, leading to a reduction of the number of episodes per month, with a lesser but positive effect on the pain intensity. Although the effects on cluster, tensiontype headaches, and on occipital neuralgia were positive, they cannot be conclusive due to the small number of patients. 


\begin{tabular}{|c|c|c|c|c|c|}
\hline Variables & \multicolumn{2}{|l|}{ Migraine ( $n=38)$} & Cluster $(\mathbf{n}=\mathbf{9})$ & other $^{c}(n=10)$ & P-Value \\
\hline Age (y) & \multicolumn{2}{|l|}{$51.79 \pm 13.67$} & $57.66 \pm 12.52$ & $55.9 \pm 12.33$ & 0.405 \\
\hline $\operatorname{BMI}\left(\mathrm{kg} / \mathrm{m}^{2}\right)$ & \multicolumn{2}{|l|}{$25.48 \pm 3.08$} & $27.63 \pm 4.43$ & $25.44 \pm 3.54$ & 0.226 \\
\hline Duration of headache $(y)$ & $20[10-344]$ & & $15[5-20]$ & $5[3-36]$ & 0.208 \\
\hline \multicolumn{6}{|l|}{$\begin{array}{l}\text { a Values are expressed as mean } \pm \text { SD or median [IQR]. } \\
\text { b Statistical tests: One-way ANOVA. } \\
{ }^{c} \text { Occipital neuralgia, tension-type headache. }\end{array}$} \\
\hline \multicolumn{2}{|l|}{ Variables } & Migraine $(\mathbf{n}=38)$ & $\operatorname{Cluster}(\mathbf{n}=\mathbf{9})$ & Other $^{c}(n=10)$ & P-Value \\
\hline \multicolumn{6}{|l|}{ Baseline } \\
\hline \multicolumn{2}{|l|}{ Number of episodes per month } & $14.5[8-25]$ & $15[15-30]$ & $16.5[15-30]$ & 0.282 \\
\hline \multicolumn{2}{|l|}{ NRS } & $8.45 \pm 1.28$ & $8.55 \pm 0.88$ & $8 \pm 1.41$ & 0.554 \\
\hline \multicolumn{2}{|l|}{ Prevention treatment (amitriptyline vs topiramate) } & 30 (78.95) vs $8(21.05)$ & $7(77.78)$ vs $2(22.22)$ & $7(70)$ vs $3(30)$ & 0.892 \\
\hline \multicolumn{2}{|l|}{ Rescue treatment (yes) } & $38(100)$ & $8(88.89)$ & $10(100)$ & - \\
\hline \multicolumn{6}{|l|}{ 1st month } \\
\hline \multicolumn{2}{|l|}{ Number of episodes per month } & $4[2-15]$ & $2[0-15]$ & $12[5-30]$ & 0.244 \\
\hline \multicolumn{2}{|l|}{ NRS } & $7[5-8]$ & $8[0-8]$ & $7[3-8]$ & 0.892 \\
\hline \multicolumn{2}{|l|}{ Prevention treatment (amitriptyline vs topiramate) } & \multicolumn{4}{|c|}{ No change compared to baseline. } \\
\hline \multicolumn{2}{|l|}{ Rescue treatment (yes) } & $35(92.11)$ & $6(66.67)$ & $9(90)$ & - \\
\hline \multicolumn{6}{|l|}{ 3rd month } \\
\hline \multicolumn{2}{|l|}{ Number of episodes per month } & $6.5[2-12]$ & $2[0-15]$ & $4.5[1-14]$ & 0.393 \\
\hline \multicolumn{2}{|l|}{ NRS } & $6.29 \pm 2.69$ & $4.55 \pm 4.09$ & $5 \pm 3.39$ & 0.217 \\
\hline \multicolumn{2}{|l|}{ Prevention treatment (amitriptyline vs topiramate) } & & No change compared t & ine. & \\
\hline Rescue treatment (yes) & & $35(92.11)$ & $4(44.44)$ & $9(90)$ & - \\
\hline 6th month & & & & & \\
\hline Number of episodes per month & & $6.5[3-12]$ & $2[1-15]$ & $4[1-15]$ & 0.774 \\
\hline NRS & & $6[5-8]$ & $8[4-8]$ & $5.5[2-8]$ & 0.564 \\
\hline Prevention treatment (amitriptyline vs topiramate) & & & No change compared tc & ine. & \\
\hline Rescue treatment (yes) & & $31(83.78)$ & $6(66.67)$ & $9(90)$ & 0.51 \\
\hline
\end{tabular}

${ }^{a}$ Values are expressed as mean \pm SD or median $[\mathrm{IQR}]$ or absolute and relative frequencies

${ }^{b}$ Statistical tests: One-way ANOVA, Kruskal-Wallis H test, Fisher's exact.

c Occipital neuralgia, tension-type headache.

PRF acts through energy released around an active electrode tip. The active tip creates a large electrical current density, producing heat of $42-44^{\circ} \mathrm{C}(1-3)$ around the nerves, modulating the synaptic signaling of the analgesic pathways, exerting its action especially on the $C$, nonmyelinated pain fibers $(7,10)$. Research suggests that PRF also modulates the early gene c-Fos $(7,11-17)$, which is responsible for the development of the second m-RNA, "preprodinorphin", of the endogenous opioid system $(1,2,11$, 13). Research proposes proposed that PRFs analgesic properties are also mediated through the noradrenergic, sero- tonergic, and endogenous opioid inhibitory pain pathways. This suggests not only peripheral but also a central modulating action (1).

The efficacy of PRF on migraines frequency was significant. Migraine is one of the most common causes of headaches, with an incidence rate of $18 \%$ in men and $43 \%$ in women (8). Although various medications have been used for its management, it often ends up refractory. Our results showed that, after PRF, the patients had less episodes of migraine per month and less intensity; and the effect lasted after 6 months post-treatment. Literature is very 


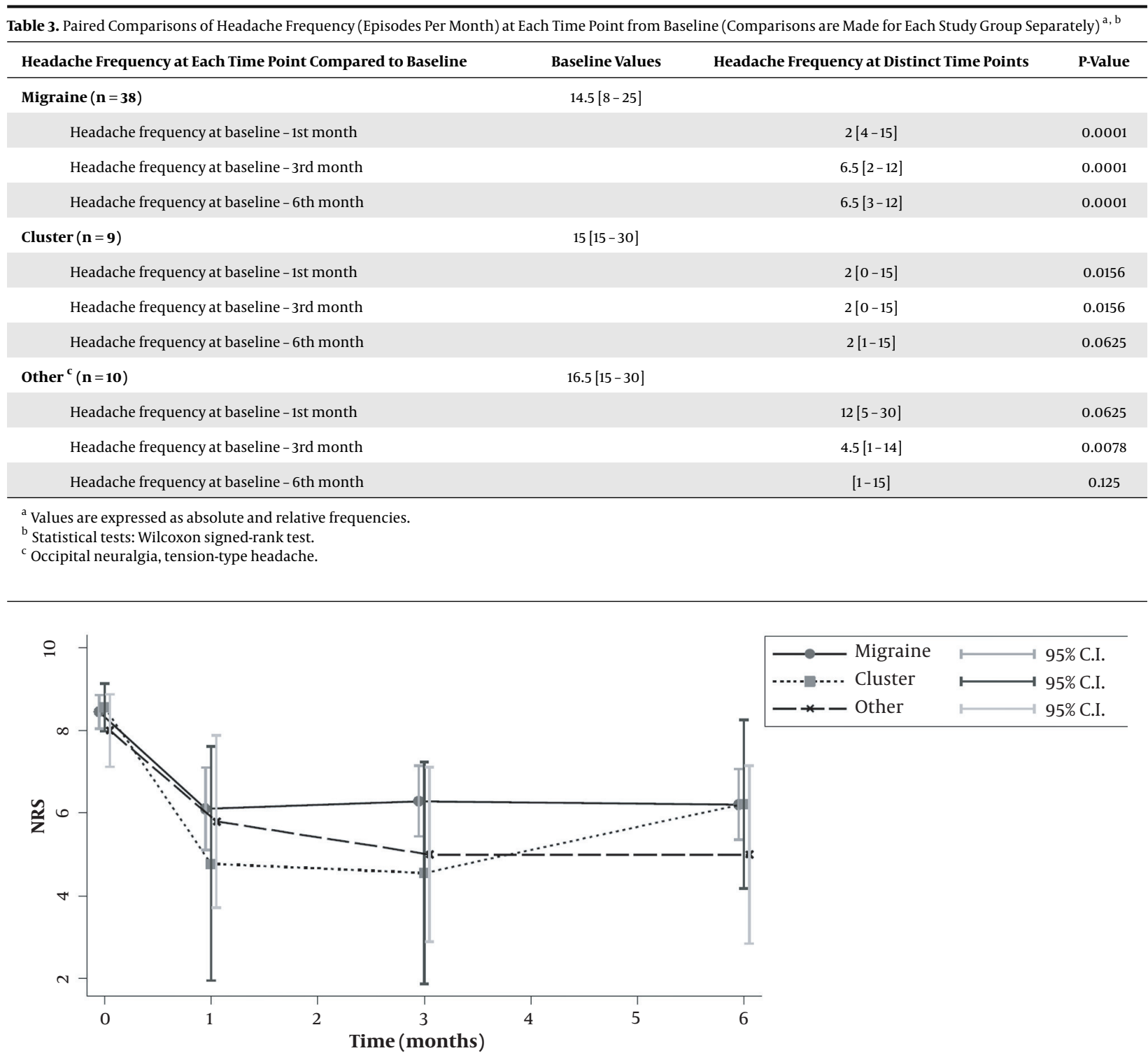

Figure 1. Diagram of headache frequency per month for each group

limited on the subject. Two case reports of PRF application for chronic migraine showed that it reduced pain intensity from 8-9 (NRS 0-10) to 3, which was sustained for 3 months postoperatively (18). Unfortunately, the time frame of follow-up was very limited in these cases, but it indicated a good short-term result. Cohen et al. (19) evaluated the effects of the technique on occipital neuralgia; their results on 45 patients with coexisting migraine showed that PRF was effective on both, occipital neuralgia and migraine. The explanation was based on the hypothesis that migraine is associated with the activation of the trigeminal and the upper cervical nerves. Nociceptive sig- nals from the fronto-oculotemporal region are transmitted via the trigeminal nerve, while pain from the occipital region is transferred through the upper cervical nerves. All these signals project centrally and converge to the trigeminal nucleus caudalis. Since GON is branch of the 2nd cervical root, its connection with the trigeminal nucleus interprets the positive effect of PRF on the control of migraine crises (18-20).

Regarding cluster, findings were also positive, with reduction of the number of episodes per month. There are no cases described in literature with application of PRF on the occipital nerves for the management of clusters, but 


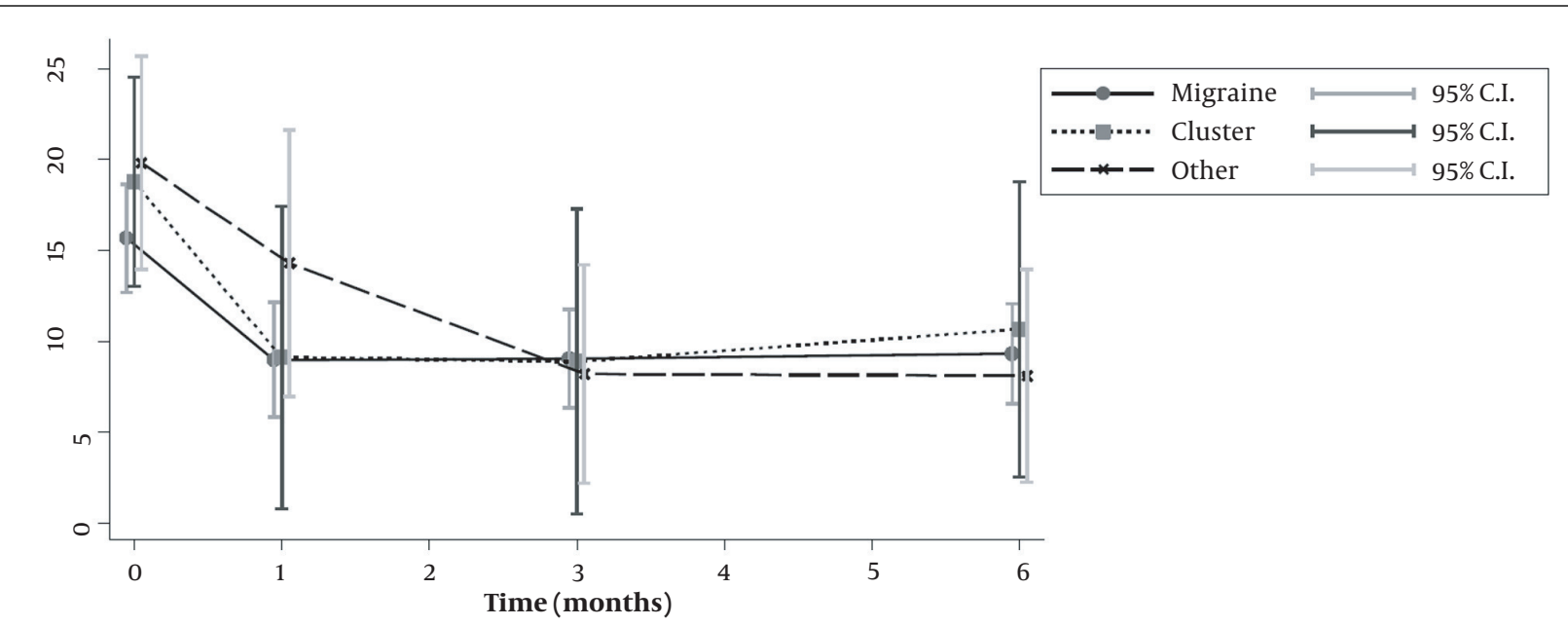

Figure 2. Diagram of pain intensity over time (numeric rating scale, NRS 0 - 10) for each group

\begin{tabular}{|c|c|c|c|}
\hline NRS Values at Each Time Point Compared to Baseline & Baseline Values & NRS at Distinct Time Points & P-Value \\
\hline Migraine $(\mathbf{n}=\mathbf{3 8})$ & $8.5[8-10]$ & & \\
\hline NRS baseline - NRS 1st month & & $7[5-8]$ & 0.0005 \\
\hline NRS baseline - NRS 3rd month & & $6[5-8]$ & 0.0001 \\
\hline Cluster $(\mathbf{n}=9)$ & $9[8-9]$ & & \\
\hline NRS baseline - NRS 1st month & & $8[0-8]$ & 0.2188 \\
\hline NRS baseline - NRS 3rd month & & $8[0-8]$ & 0.0313 \\
\hline NRS baseline - NRS 6th month & & $8[4-8]$ & 0.0625 \\
\hline $\operatorname{other}^{c}(n=10)$ & $8[7-9]$ & & \\
\hline NRS baseline - NRS 1st month & & $7[3-8]$ & 0.125 \\
\hline NRS baseline - NRS 3rd month & & $4.5[4-8]$ & 0.0156 \\
\hline NRS baseline - NRS 6th month & & $5.5[2-8]$ & 0.0625 \\
\hline
\end{tabular}

${ }^{a}$ Values are expressed as absolute and relative frequencies.

${ }^{\mathrm{b}}$ Statistical tests: Wilcoxon signed-rank test.

${ }^{c}$ Occipital neuralgia, tension-type headache.

only PRF of the sphenopalatine ganglion. However, there are various published case series indicating that injection of high-volume local anesthetic with corticosteroid on the occipital nerves at the side of the cluster may lead to a preventive effect $(18,20-23)$. That is the reason we decided to proceed to PRF of the occipital nerves for a more sustained result. However, more patients are required to confirm this effect.

Tension-type headache and occipital neuralgia were also treated with PRF with positive results; however, in our study, the number of patients to prove such an effect was limited. Literature is conflicting about tension type and cervicogenic headaches regarding occipital nerve blocks, while PRF has not been studied. Hascalovici \& Robbins (24) reported a positive effect of $67 \%$ of occipital nerve blocks for tension-type headaches. Gabrhelík et al. (25) performed PRF of GON in 15 patients leading to a successful outcome for a period of 9 months, a finding that supports our results. Also, regarding occipital neuralgia, several previous studies reported that the PRF stimulation on the occipital nerves may be effective (26-30). In their review study, Manolitsis and Elahi (26) reported promising results, including a total of three clinical studies and one case report. Improvements in pain, quality of life, and adjuvant pain medication consumption were demonstrated, a fact that requires further investigation. Certainly, more studies are required 


\begin{tabular}{|c|c|c|c|c|}
\hline Variables & Migraine $(n=38)$ & Cluster $(\mathbf{n}=9)$ & other $^{c}(n=10)$ & P-Value \\
\hline \multicolumn{5}{|l|}{ Rescue treatment at baseline } \\
\hline No meds $|<3| \geq 3$ meds & $0 / 32(84.21) / 6(15.79)$ & $1(11.11) / 8(88.89) / 0$ & $0 / 8(80) / 22(20)$ & 0.21 \\
\hline Paracetamol (yes) & $19(50)$ & $5(55.55)$ & $9(90)$ & \\
\hline Triptans (yes) & $25(65.79)$ & $3(33.33)$ & $2(20)$ & \\
\hline Opioids (yes) & $8(21.05)$ & $1(11.11)$ & $1(10)$ & \\
\hline \multicolumn{5}{|l|}{ Rescue treatment at 1st month } \\
\hline No meds $\mid<3 / \geq 3$ meds & $3(7.89) / 31(81.58) / 4(10.53)$ & $3(33.33) / 5(55.56) / 1(11.11)$ & $1(10) / 7(70) / 2(20)$ & 0.195 \\
\hline Paracetamol (yes) & $16(42.11)$ & $4(44.44)$ & $8(80)$ & \\
\hline NSAIDs (yes) & $16(42.11)$ & $3(33.33)$ & $4(40)$ & \\
\hline Triptans (yes) & $20(52.63)$ & $2(22.22)$ & $2(20)$ & \\
\hline Opioids (yes) & $6(15.79)$ & $1(11.11)$ & $1(10)$ & \\
\hline \multicolumn{5}{|l|}{ Rescue treatment at 3rd month } \\
\hline No meds $|<3| \geq 3$ meds & $4(10.53) / 31(81.58) / 3(7.89)$ & $5(55.56) / 3(33.33) / 1(11.11)$ & $1(10) / 7(70) / 2(20)$ & 0.015 \\
\hline Paracetamol (yes) & $15(39.47)$ & $3(33.33)$ & $7(70)$ & \\
\hline NSAIDs (yes) & $15(39.47)$ & $2(22.22)$ & $3(30)$ & \\
\hline Triptans (yes) & $19(50)$ & $1(11.11)$ & $2(20)$ & \\
\hline Opioids (yes) & $6(15.79)$ & $1(11.11)$ & $2(20)$ & \\
\hline \multicolumn{5}{|l|}{ Rescue treatment at 6th month } \\
\hline No meds $\mid<3 / \geq 3$ meds & $7(18.42) / 28(73.68) / 3(7.89)$ & $3(33.33) / 5(55.56) / 1(11.11)$ & $1(10) / 7(70) / 2(20)$ & 0.521 \\
\hline NSAIDs (yes) & $13(34.21)$ & $3(33.33)$ & $3(30)$ & \\
\hline Triptans (yes) & $18(47.37)$ & $1(11.11)$ & $3(30)$ & \\
\hline Opioids (yes) & $6(15.79)$ & $1(11.11)$ & $2(20)$ & \\
\hline
\end{tabular}

${ }^{\text {a }}$ Values are expressed as absolute and relative frequencies.

b Statistical tests: Fisher's exact.

${ }^{\text {c }}$ Occipital neuralgia, tension-type headache.

to further assess this effect and possibly relate it to the exact mode of action of PRF.

The main limitation of this study is the absence of randomization of patients with another form of treatment. However, this could not be performed due to financial constraints. This is why we chose to perform a case series study to present our results. Another limitation is the small sample of cluster headaches and other forms of headaches; however, these results represent the real statistics of headache clinic prevalence, being mostly patients with chronic migraine.

The results of this study showed a strong positive effect of PRF of the occipital nerves on chronic headache management, mostly reducing the frequency of headache crises per month. This result should be further evaluated in future studies with longer follow-up period to assess the long-term efficacy.

\section{Footnotes}

Authors' Contribution: Study concept and design, Batistaki C, Madi A, and Arvaniti C; Acquisition of data, Batistaki C, Madi A, Karakosta A, Arvaniti C.; Analysis and interpretation of data, Batistaki C, Madi A, Arvaniti C, Karakosta A.; Drafting of the manuscript, Batistaki C, Madi A.; Critical revision of the manuscript for important intellectual content, Batistaki C, Madi A, Karakosta A, Kostopanagiotou G, Arvaniti C.; Statistical analysis, Karakosta A.; Administrative, technical, and material support, University Hospital Attikon; Study supervision, Batistaki C, Kostopanagiotou G.

Clinical Trial Registration Code: NCT04339335

Conflict of Interests: There were no conflicts of interest. 


\section{Ethical Approval:}

896/29-01-2019;

\section{http://www.attikonhospital.gr/.}

Funding/Support: There was no funding for this study. The research was performed in a university hospital setting.

Informed Consent: Written informed consent was obtained from all participants.

\section{References}

1. Chua NH, Vissers KC, Sluijter ME. Pulsed radiofrequency treatment in interventional pain management: mechanisms and potential indications-a review. Acta Neurochir (Wien). 2011;153(4):763-71. doi: 10.1007/s00701-010-0881-5. [PubMed: 21116663]. [PubMed Central: PMC3059755].

2. Bogduk N. Pulsed radiofrequency. Pain Med. 2006;7(5):396-407. doi: 10.1111/j.1526-4637.2006.00210.x. [PubMed: 17014598].

3. Van Zundert J, Patijn J, Hartrick C, Lataster A, Huygen F, Mekhail N, et al. Evidence-based interventional pain medicine: according to clinical diagnoses. New Jersey, USA: John Wiley \& Sons; 2011.

4. Narouze SN. Interventional management of head and face pain. New York, UK: Springer Science; 2014. p. 59-60.

5. Vanneste T, Van Lantschoot A, Van Boxem K, Van Zundert J. Pulsed radiofrequency in chronic pain. Curr Opin Anaesthesiol. 2017;30(5):57782. doi: 10.1097/ACO.0000000000000502. [PubMed: 28700369].

6. Gooriah R, Nimeri R, Ahmed F. Evidence-based treatments for adults with migraine. Pain Res Treat. 2015;2015:629382. doi: 10.1155/2015/629382. [PubMed: 26839703]. [PubMed Central: PMC4709728].

7. Byrd D, Mackey S. Pulsed radiofrequency for chronic pain. Curr Pain Headache Rep. 2008;12(1):37-41. doi: 10.1007/s11916-008-0008-3. [PubMed: 18417022]. [PubMed Central: PMC2913603].

8. Tepper SJ. Advanced interventions for headache. Headache. 2012;52 Suppl 1:50-4. doi: 10.1111/j.1526-4610.2012.02133.x. [PubMed: 22540208].

9. Abejon D, Reig E. Is pulsed radiofrequency a neuromodulation technique? Neuromodulation. 2003;6(1):1-3. doi: 10.1046/j.15251403.2003.03009.x. [PubMed: 22150906].

10. Brasil LJ, Marroni N, Schemitt E, Colares J. Effects of pulsed radiofrequency on a standard model of muscle injury in rats. Anesth Pain Med. 2020;10(1). e97372. doi: 10.5812/aapm.97372. [PubMed: 32309197]. [PubMed Central: PMC7144246].

11. Van Zundert J, de Louw AJ, Joosten EA, Kessels AG, Honig W, Dederen PJ, et al. Pulsed and continuous radiofrequency current adjacent to the cervical dorsal root ganglion of the rat induces late cellular activity in the dorsal horn. Anesthesiology. 2005;102(1):125-31. doi: 10.1097/00000542-200501000-00021. [PubMed: 15618796].

12. Hunter JC, Woodburn VL, Durieux C, Pettersson EK, Poat JA, Hughes J. c-fos antisense oligodeoxynucleotide increases formalin-induced nociception and regulates preprodynorphin expression. Neuroscience. 1995;65(2):485-92. doi: 10.1016/0306-4522(94)00500-5. [PubMed: 7777163].

13. Arvaniti C, Madi AI, Kostopanagiotou G, Batistaki C. Can pulsed radiofrequency of the occipital nerves cause sedation? A new perspective of existing knowledge. Anesth Pain Med. 2020;10(2). e96418. doi: 10.5812/aapm.96418. [PubMed: 32754427]. [PubMed Central: PMC7352942].

14. Sluijter ME, Imani F. Evolution and mode of action of pulsed radiofrequency. Anesth Pain Med. 2013;2(4):139-41. doi: 10.5812/aapm.10213. [PubMed: 24223349]. [PubMed Central: PMC3821144].

15. Imani $\mathrm{F}$, Gharaei $\mathrm{H}$, Rezvani M. Pulsed radiofrequency of lumbar dorsal root ganglion for chronic postamputation phantom pain. Anesth
Pain Med. 2012;1(3):194-7. doi: 10.5812/kowsar.22287523.3768. [PubMed: 24904793]. [PubMed Central: PMC4018701].

16. Imani F. Using pulsed radiofrequency for chronic pain. Anesth Pain Med. 2012;1(3):155-6. doi: 10.5812/kowsar.22287523.4047. [PubMed: 24904784]. [PubMed Central: PMC4018683].

17. Kwak S, Chang MC. Management of refractory chronic migraine using ultrasound-guided pulsed radiofrequency of greater occipital nerve: Two case reports. Medicine. 2018;97(45). e13127. doi: 10.1097/MD.0000000000013127. [PubMed: 30407333]. [PubMed Central: PMC6250499].

18. Gonen M, Balgetir F, Aytac E, Tasci I, Demir CF, Mungen B. Suboccipital steroid injection alone as a preventive treatment for cluster headache. J Clin Neurosci. 2019;68:140-5. doi: 10.1016/j.jocn.2019.07.009. [PubMed: 31326284].

19. Cohen SP, Peterlin BL, Fulton L, Neely ET, Kurihara C, Gupta A, et al. Randomized, double-blind, comparative-effectiveness study comparing pulsed radiofrequency to steroid injections for occipital neuralgia or migraine with occipital nerve tenderness. Pain. 2015;156(12):2585-94. doi: 10.1097/j.pain.0000000000000373. [PubMed: 26447705]. [PubMed Central: PMC4697830].

20. Rozen TD. High-volume anesthetic suboccipital nerve blocks for treatment refractory chronic cluster headache with long-term efficacy data: An observational case series study. Headache. 2019;59(1):5662. doi: 10.1111/head.13394. [PubMed: 30144049].

21. Gaul C, Roguski J, Dresler T, Abbas H, Totzeck A, Gorlinger K, et al. Efficacy and safety of a single occipital nerve blockade in episodic and chronic cluster headache: A prospective observational study. Cephalalgia. 2017;37(9):873-80. doi: 10.1177/0333102416654886. [PubMed: 27313215].

22. Lambru G, Abu Bakar N, Stahlhut L, McCulloch S, Miller S, Shanahan $P$, et al. Greater occipital nerve blocks in chronic cluster headache: A prospective open-label study. Eur J Neurol. 2014;21(2):338-43. doi: 10.1111/ene.12321. [PubMed: 24313966].

23. Amighi D, Majedi H, Tafakhori A, Orandi A. The efficacy of sphenopalatine ganglion block and radiofrequency denervation in the treatment of cluster headache: A case series. Anesth Pain Med. 2020;10(6). e104466. doi: 10.5812/aapm.104466. [PubMed: 34150572]. [PubMed Central: PMC8207843].

24. Hascalovici JR, Robbins MS. Peripheral nerve blocks for the treatment of headache in older adults: A retrospective study. Headache. 2017;57(1):80-6. doi: 10.1111/head.12992. [PubMed: 27901275].

25. Gabrhelik T, Michalek P, Adamus M. Pulsed radiofrequency therapy versus greater occipital nerve block in the management of refractory cervicogenic headache - a pilot study. Prague Med Rep. 2011;112(4):27987. [PubMed: 22142523].

26. Manolitsis N, Elahi F. Pulsed radiofrequency for occipital neuralgia. Pain Physician. 2014;17(6):E709-17. [PubMed: 25415786].

27. Huang JH, Galvagno SM, Hameed M, Wilkinson I, Erdek MA, Patel A, et al. Occipital nerve pulsed radiofrequency treatment: A multi-center study evaluating predictors of outcome. Pain Med. 2012;13(4):489-97. doi: 10.1111/j.1526-4637.2012.01348.x. [PubMed: 22390409].

28. Navani A, Mahajan G, Kreis P, Fishman SM. A case of pulsed radiofrequency lesioning for occipital neuralgia. Pain Med. 2006;7(5):453-6. doi: 10.1111/j.1526-4637.2006.00217.x. [PubMed: 17014606].

29. Stall RS. Noninvasive pulsed radio frequency energy in the treatment of occipital neuralgia with chronic, debilitating headache: A report of four cases. Pain Med. 2013;14(5):628-38. doi: 10.1111/pme.12078. [PubMed: 23566238].

30. Vanderhoek MD, Hoang HT, Goff B. Ultrasound-guided greater occipital nerve blocks and pulsed radiofrequency ablation for diagnosis and treatment of occipital neuralgia. Anesth Pain Med. 2013;3(2):2569. doi: 10.5812/aapm.10985. [PubMed: 24282778]. [PubMed Central: PMC3833045]. 